\title{
Design and Implementation of Substation Operation Safety Monitoring and Management System Based on Three- Dimensional Reconstruction
}

\author{
Yingyi YANG ${ }^{\mathrm{a}, \mathrm{b}, 1}$, Hao WU, ${ }^{\mathrm{a}, \mathrm{b}}$, Fan $\mathrm{YANG}^{\mathrm{c}}$, Xiaoming MAI ${ }^{\mathrm{a}, \mathrm{b}}$ and Hui CHEN ${ }^{\mathrm{a}, \mathrm{b}}$ \\ ${ }^{a}$ Guangdong Diankeyuan Energy Technology Co., Ltd. \\ ${ }^{\mathrm{b}}$ Electric Power Research Institute of Guangdong Power Grid Co., Ltd. \\ ' Shaoguan Power Supply Bureau of Guangdong Power Grid Co., Ltd.
}

\begin{abstract}
In order to reduce operational risks and to improve the risk management and control level in substation, a substation operation safety monitoring and management system $(3 \mathrm{D} 2 \mathrm{~S} 2 \mathrm{M})$ has been structured based on three-dimensional (3D) laser modeling technology. In this paper, we introduce how to build such a system and to describe its implementation details. A 3D lidar scanning technology is used to perform a holographic scan of the whole internal area in a substation to obtain color point cloud data of buildings and all equipment. Then, a novel 3D visualization safety monitoring and management system, named $3 \mathrm{D} 2 \mathrm{~S} 2 \mathrm{M}$, is developed by performing a $3 \mathrm{D}$ reconstruction of the point cloud data. Based on the real 3D scene model of 3D2S2M, the method of 3D distance measurement is used to replace manual on-site investigation for improving operation and maintenance efficiency. In addition, a real-time high-accuracy localization method is proposed, in order to identify and analyze the positioning and the behavior of the personnel, and the movement trajectory of the equipment. By combining positioning information and the electronic fence that used in 3D2S2M, risk levels of the personnel (or equipment) are evaluated and the corresponding alarm is issued to prevent dangerous behavior, thereby the operation risk is reduced in substation.
\end{abstract}

Keywords. substation, safety monitoring and management, three-dimensional reconstruction, personnel localization

\section{Introduction}

Substations are key nodes in the power grid. Their safety and stability greatly influence the whole electric power system. With the continuous development of smart substations [1], a large number of new equipment and increasingly complex building structures in substations have made the intelligent substations present the following new characteristics in substation safety monitoring and management: First, the introduction of the concept of regionalization and hierarchical security makes the construction of substations tend to be functional, which makes some safety management in the substation, such as the joint security dispatch of cyber-physical systems, remote supervision, and security dispatch, more difficult. Specifically, under the concept of regionalized

\footnotetext{
${ }^{1}$ Corresponding Author. Email: yangyingyi10@126.com
} 
management, the traditional on-site investigation methods and manual dispatching methods in substation are with high security risks, multi-inspection points, and multidangerous points. Moreover, traditional inspection carried out artificially is complicated and time-consuming [2-3]. The predictive maintenance is difficult to implement, which makes it difficult to achieve special response strategies formulated by potential problem equipment or conditions. In addition, some special areas are difficult to supervise and personnel safety is difficult to guarantee. Second, safety monitoring and management of substations are faced with huge challenges due to the diverse operating environment and the complex composition of operating personnel. Everyone knows that traditional safety management processes place operating personnel at greater personal risks. And now, in the intelligent substation, diverse operating environment will make the operators with different job responsibilities work in different operating areas. In this case, it is difficult to know accurately whether the personnel are on the job, and there is a risk of accidents resulting in personal injury or death caused by misoperation. In addition, complex personnel entering and leaving will also make a huge threat to the safety of equipment in the operation inspection area. All these reasons can bring challenges to traditional security management.

Personnel positioning is a very essential issue in substation safety management. In some power facility monitoring systems that adopt some simple image processing algorithms, the monitoring of personnel targets stays in a situation where a small number of targets are resolved and classification is easy, and once a personnel target is detected, an alarm is issued to him in any case. It is obviously not reasonable, and these methods cannot monitor the behavior of personnel targets [4].

Therefore, there is an urgent need to develop a new safety monitoring and management system that can simultaneously control risk, prevent violations and avoid accidents. Virtual visualization technology based on three-dimensional reconstruction has become an effective solution to these problems [5].

\section{Related Work}

In recent years, three-dimensional real scene reconstruction of substation has been becoming one of the most popular techniques in intelligent substation safety management [6-7]. It mainly includes: geometric structure modeling method, VRM modeling method [8] and laser scanning modeling method [9]. Different methods have different principles, and their performance and application areas are also different [10]. Quintana $\mathrm{J}$ et al. [11] developed a substation simulation training system based on the geometric shapes of different electrical equipment. Wang et al. [12] enriched the electrical equipment material model based on the virtual reality technology to complete the 3D modeling of the substation, and added scene special effects, defect simulations, and other functions. The geometric structure modeling method does not need to separately model different electrical equipment, and can directly select a specific geometric structure, which greatly improves the modeling efficiency and the overall reconstruction effect, but the reconstruction precision is not high, and it is mostly used for simulation training [13]. Li [14] used a 3D laser scanner to scan the substation, and used an improved particle swarm algorithm to complete the automatic identification and classification of the $3 \mathrm{D}$ point cloud, and finally realized the $3 \mathrm{D}$ reconstruction of the substation, which improved the reconstruction efficiency and precision. Du et al. [15] also used a 3D laser scanner to scan a substation to obtain a 3D point cloud, and used 
virtual simulation technology to simulate the staff's inspection and maintenance of electrical equipment in a reconstructed 3D scene. This method of acquiring and reconstructing a $3 \mathrm{D}$ point cloud of a substation by a 3D laser scanner has high reconstruction precision, accurate $3 \mathrm{D}$ positioning, and high degree of detail reduction of electrical equipment, and is suitable for practical engineering applications such as site monitoring, positioning, and measurement of a substation.

In order to improve the substation operation safety risk management level and to reduce the operation risk, accurate positioning of activity targets in the substation should be implemented in a substation operation safety monitoring and management system [16]. At present, in terms of substation personnel (or machine) safety monitoring, Zhou et al. [17] combined Zigbee positioning technology with fuzzy inference algorithm for realtime positioning of field workers. But this method has low positioning accuracy and is not convenient for the actual operation. Huang [18] proposed an ultrasonic orthogonal spread-spectrum coding technology based on pseudo-random modulation carrier, and built a precise positioning model for the staff in the substation. This method locates accurately. But the accuracy decays rapidly due to the decay of the ultrasonic wave propagation. Although the positioning interval is good, the accuracy is greatly reduced, so it is only suitable for positioning in specific scenarios of substations [18]. Yuan et al. [19] installed wireless sensor network (WSN) nodes in key areas of the substation to receive signals in real time, and completed background real-time location monitoring of field workers through signal conversion. However, this method has high equipment costs, and the monitoring methods still have a certain degree of lag. Zhang et al. [20] used a multi-module and multi-frequency GNSS (Global Navigation Satellite System) module equipped with GPS (Global Positioning System) or Beidou, and adopted high-precision outdoor differential positioning technology to complete real-time positioning for substation staff which has higher positioning accuracy. Du et al. [21] used a binocular stereo vision camera combined with an inertial measurement device to complete realtime positioning and predictive tracking of workers.

\section{The Framework of 3D2S2M}

The new 3D2S2M uses a 3D laser scanning technology to perform a holographic scan of the entire substation to obtain high-precision three-dimensional color point cloud data [22]. Through 3D reconstruction and importing the equipment ledger data, a 3D visualization platform for substation safety monitoring and management is constructed. Meanwhile, multiple related functional modules are developed based on the 3D visualization platform. For example, the virtual on-site survey module is developed to replace the inefficient and high-risk manual on-site investigation. The high-precise realtime localization module for personnel (or machine) are designed to intelligently identify and analyze the behavior of personnel and the trajectory of equipment in the substation, and thus the level of risk management and control of substation are improved. In risk level assessment and real-time alarm module, based on the data of equipment ledger and safety live distance, combined with the other data, such as positioning data of operator, vehicle or equipment, positioning delay, motion trajectory and inertia parameters , a corresponding motion model is established and appropriate operational risk early warning strategies are formulated. It is worth noting that the interface module is 
responsible for data exchange between the new system and all external systems. The overall framework of the new system is shown in Figure 1.

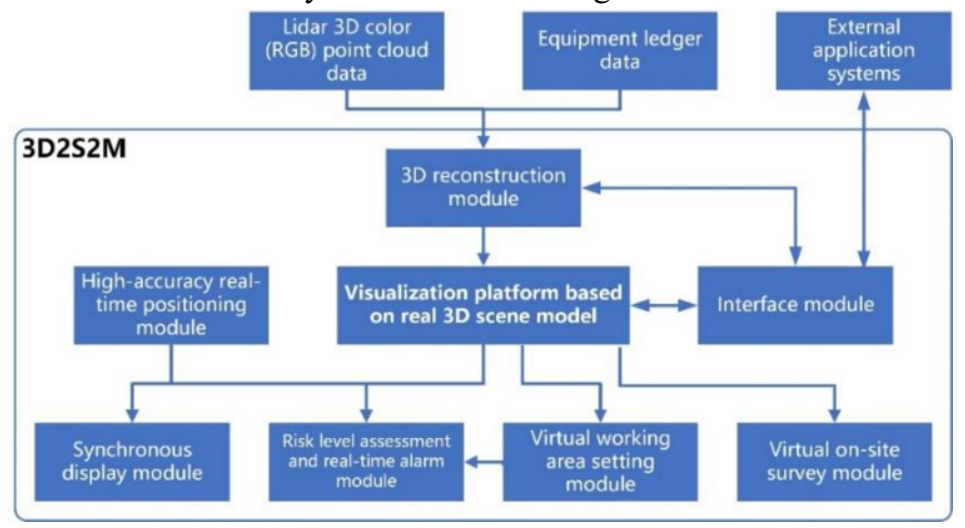

Figure 1. The framework of 3D2S2M.

\section{Implementation of 3D2S2M}

\subsection{Three-dimensional Real Scene Reproduction of Substation}

Modeling precision is the decisive factor for reconstructing three-dimensional real scene of the whole substation. To gather the $3 \mathrm{D}$ color (RGB) point cloud data with precise coordinates for all objects in substation, this new system adopts high-precision 3D lidar scanning device (error is $\pm 2 \mathrm{~cm}$ ) to perform a full-substation mobile scanning. Through the holographic scanning for all objects in the substation, including various electrical equipment, site layout, actual structure, and so on, the $3 \mathrm{D}$ scene of the substation is accurately reconstructed based on the spatial coordinate information of latitude, longitude, height, and depth. The specification of lidar sensor used is listed in Table 1.

Table 1. The specification of lidar sensor used.

\begin{tabular}{cc}
\hline Parameters & Specifications \\
\hline range type & phase \\
laser class & 1R eye-safe laser \\
scan scope & 150 meters \\
range error & $\pm 1 \mathrm{~mm}$ \\
range noise & $\leq 0.3 \mathrm{~mm}$ within 25 meters \\
field angle & vertical $300^{\circ}$, horizontal $360^{\circ}$ \\
\hline
\end{tabular}

The core technology of the 3D2S2M is the high-precision reconstruction of the real scene inside the substation, including the coordinates, size and even the operating conditions of equipment. Therefore, the ID of the 3D virtual device in the 3D2S2M must be the same as the ID number of the real device in the substation. That is to say, 3D virtual devices need to be bound to the actual device by using the same ID to access the equipment ledger data and monitoring data. Equipment ledger data are inserted into the 3D model using offline copying method, so that the ledger information can be displayed intuitively in 3D2S2M. 


\subsection{Measurement Analysis in Three-dimensional Space for Virtual On-site Survey}

The on-site investigation in substation consumes large costs, such as manpower and material resources. Multiple on-site investigations before the maintenance operation would be time consuming and inefficient.

In the 3D2S2M, virtual on-site investigation can be performed to replace the on-site Investigation: measurement tools in the 3D2S2M are used to obtain 3D spatial distance parameters (including manual measurements, air-ground ranging,) to achieve the goals of field inspection and measurement, and then make accurate assessments and decisions about field operations. Accurate spatial distance information can also be used to evaluate and calculate various on-site operations, which will greatly improve the work efficiency.

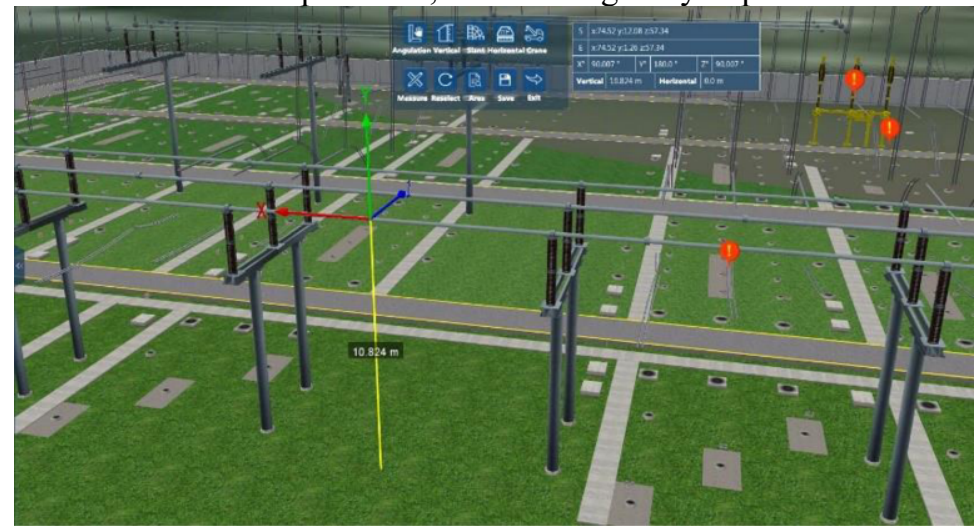

Figure 2. Illustration of distance measurement.

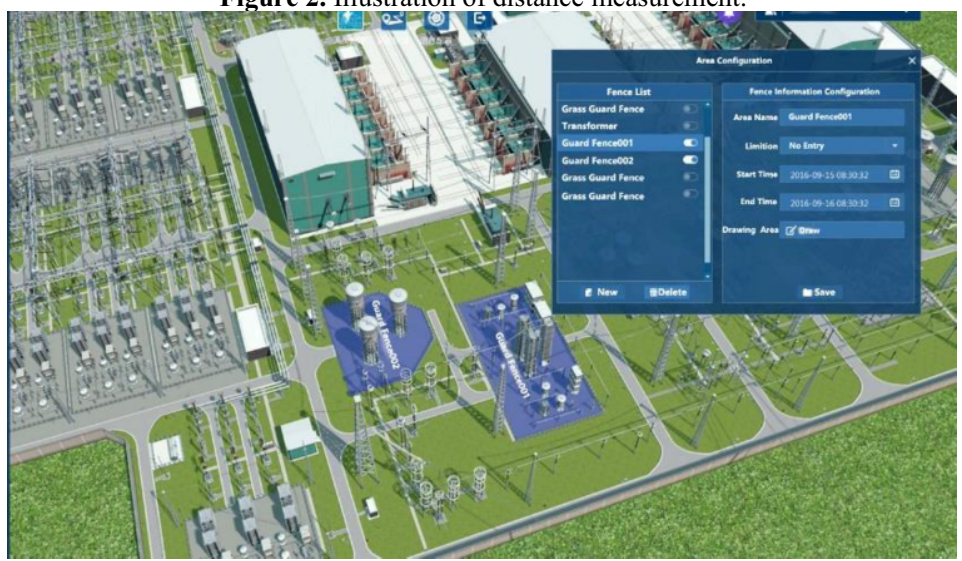

Figure 3. Illustration of the virtual working area setting based on three-dimensional electric fence.

For example, when ranging in our three-dimensional scene, let $S$ be the starting point and $\boldsymbol{E}$ be the end point. In the 3D2S2M's 3D scene, the values of their $\boldsymbol{X}, \boldsymbol{Y}$, and $\boldsymbol{Z}$ coordinate axes will be displayed accordingly. Our angle of $\boldsymbol{X}$ ( or $\boldsymbol{Y}$, or $\boldsymbol{Z}$ ) is defined as the angle between the line connecting these two points and the $\boldsymbol{X}$ axis (or $\boldsymbol{Y}$, or $\boldsymbol{Z}$ ). The distance between the two points is defined as the straight-line distance between the two points. Moreover, the horizontal distance is defined as the distance between the two points in the same horizontal direction. Figure 2 shows an example of distance measurement in $3 \mathrm{D} 2 \mathrm{~S} 2 \mathrm{M}$. The measurement result is $10.824 \mathrm{~m}$ in this example. 


\subsection{Setting up the Virtual Working Area}

The 3D2S2M sets the virtual working area by setting a 3D virtual electric fence. According to the characteristics of the operation, the electric fence is divided into [No Entry], [Ban Out], [Live Equipment] and other types. At the same time, these areas can be named, and its period of validity and the violation contents can also be set, such as illegal stay time, out time, safety distance, and so on. A virtual working area is illustrated as Figure 3.

\subsection{High-accuracy Personnel (or Equipment) Positioning}

\subsubsection{Calculating the High-accuracy Coordinates of Personnel (or Equipment)}

In this paper, the high- accuracy positioning of personnel (or equipment) in the 3D scene is achieved by using the data gathered from the GPRS (General Packet Radio Service) mobile terminal with a high-accuracy positioning device. The geographic location information obtained by the GPS receiving module is transmitted to the server (i.e. base station) through the GPRS wireless communication network, and processed on the server to achieve remote positioning [23]. Data accuracy of the high-accuracy positioning device in the GPRS mobile terminal can reach the centimeter level. The RTK differential base station (BS) receives the GPS + GLONASS (GLOBAL NAVIGATION SATELLITE SYSTEM) + BeiDou satellite signal, then decodes them by using the algorithm stored in its MCU1, and outputs RTK differential signal in RTCM format. The mobile terminal receives the GPS + GLONASS satellite signals and the RTK differential signals from the BS, and decodes them by using the algorithm stored in its MCU2. The whole positioning process can be summarized as follows: Firstly, calculating the known precise coordinates of the base station by using the following formula:

$$
\tilde{\rho}_{R i}^{j}=\rho_{R i}^{j}+c \delta t_{i}+d_{i o n}+d_{\text {trop }}+\varepsilon_{\rho}
$$

where $\tilde{\rho}_{R i}^{j}$ is the precise coordinates, ${ }^{j}{ }_{R i}^{j}$ is the measured value of the coordinates, $c \delta t_{i}$ is the satellite clock error, $d_{i o n}$ is the ionospheric error, ${ }_{t r o p}$ is the tropospheric error and $\varepsilon_{\rho}$ is coordinates of the base station.

Secondly, the error from the base station to the satellite is calculated by the following formula:

$$
\Delta P_{\text {err }}=\tilde{\rho}_{R i}^{j}-\rho_{R i}^{j}=c \delta t_{i}+d_{\text {ion }}+d_{\text {trop }}+\varepsilon_{\rho}
$$

At last, the mobile terminal corrects its localization result by receiving the error from BS to improve its positioning accuracy by the formula:

$$
\tilde{\rho}_{M i}^{j}=\rho_{M i}^{j}+\Delta P_{\text {err }}=\rho_{M i}^{j}+c \delta t_{i}+d_{i o n}+d_{\text {trop }}+\varepsilon_{\rho}
$$


where $\rho_{M i}^{j}$ is the measured coordinates of the target, and $\tilde{\rho}_{M i}^{j}$ is the corrected coordinates of the target.

\subsubsection{Locating Personnel (or Equipment) in 3D Scene}

In a $3 \mathrm{D}$ scene, the longitude and latitude of the ordinate origin is used as the reference, and the coordinates of the target (personnel or equipment) in the scene are converted from their latitude and longitude.

Let $O$ be the origin of the coordinates, its longitude and latitude are $O_{l a}$ and $O_{l o}$ respectively, and its coordinates in the 3D scene are $(0,0,0)$; the corrected longitude and latitude of the target $(M)$ are $M_{l a}, M_{l o}$, its coordinates in 3D scene are $(M x, M y, M z(=0))$. Then, the $x$ and $y$ coordinates of $M$ can be calculated by the following formula:

$$
\begin{aligned}
& M x=\left(M_{l a}-O_{l a}\right) \times K 1 \\
& M y=\left(M_{l o}-O_{l o}\right) \times K 2
\end{aligned}
$$

where $K 1$ and $K 2$ are the magnifications of the $x$-axis and $y$-axis of the 3D scene and the real scene, respectively.

For working equipment with a height such as a crane, the height must be marked by tools such as laser detection and image recognition. The localization results of personnel and equipment are shown in Figure 4.

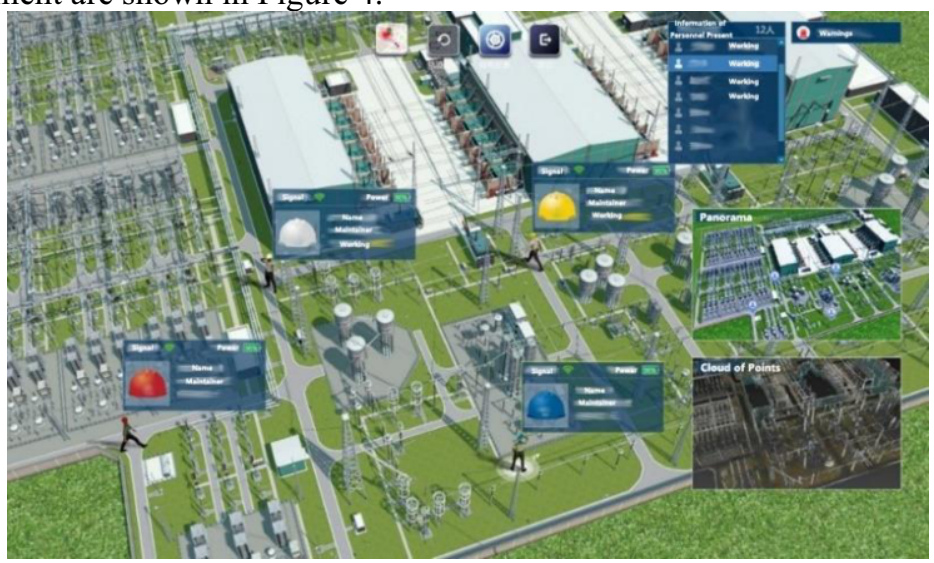

Figure 4. Illustration of positioning of personnel and equipment.

In addition, based on the real-time positioning data of the target, the $3 \mathrm{D} 2 \mathrm{~S} 2 \mathrm{M}$ can support the identity binding and real-time positioning of the on-site personnel (or equipment). Meanwhile, it also supports the review of the historical trajectory of personnel movement, and the viewing of the specific information and alarm records of a node in the historical trajectory can facilitate work performance assessment and security risk assessment.

High-accuracy positioning coordinates can be mapped to the 3D scene accurately and presented visually by adopting our satellite positioning method. Here satellite positioning we adopted is compared with other major methods, including UWB (Ultra Wide Band), RFID (Radio Frequency Identification) and WIFI. Table 2 shows the comparison among those methods for substations in terms of accuracy, cost, stability and real-time. 
Table 2. The comparison among satellite, UWB, RFID and WIFI.

\begin{tabular}{ccccc}
\hline Methods & Accuracy & Cost & Stability & Real-time \\
\hline satellite & centimetre level & relatively lower & good & good \\
decimeter level/ & higher & relatively stable & good \\
UWB & centimetre level & & relatively stable & good \\
RFID & decimeter level & very low & poor & poor \\
WIFI & decimeter level & relatively lower & .
\end{tabular}

As shown in Table 2, in the situation of cruel electromagnetism environment in the substations, satellite positioning method we adopted achieves centimetre level, the best one among all the methods. Although UWB method presents the level close to that of satellite method, it costs more. As for RFID and WIFI method, both have lower accuracy. RFID has poor real-time performance while WIFI has poor stability.

\subsection{Assessing Risk Levels and Real-time Alerts}

3D2S2M aims at the safety monitoring and management of the on-site operation region in substations. The new system performs big data statistical analysis and historical risk data analysis, and assesses the risk level based on the movement trajectory of personnel and equipment.

Using historical big data about massive filed tasks, 3D2S2M statistically analyzes personnel path trajectories and working areas based on positioning coordinates. The best path trajectory and safe working area for a specific task can then be predicted with Markov algorithm based on statistical analysis results. The system classifies the potential risks in actual execution, including too close distance between personnel and equipment, straying into non-working areas, diverging from the normal trajectory and sticking around abnormally for a long time, from highest to lowest priority and taking layered pre-warning accordingly. Besides, clustering analysis on coordinates and trajectory similarity comparison are also adopted by $3 \mathrm{D} 2 \mathrm{~S} 2 \mathrm{M}$ for hotspot judgment, thus finding abnormal working places and raising alarm in time.

For high-risk and accident-prone locations, visually display the heat map of the working area in our 3D scene, and part of them are displayed as the work blind zone. By analyzing personnel and equipment movement trajectory in combination with virtual electronic fences, equipment operating data, our 3D2S2M can dynamically judge operating risks and trigger alarms in time. Besides, the 3D2S2M can also realize the synchronization display of the movements (attitudes) of large machine (such as cranes used in field operations, baskets, boom frames, and boom frames of curved arm climbing car) with vehicle real $3 \mathrm{D}$ scene models (such as cranes). Therefore, the 3D2S2M can evaluate the risk of triggering operations by dynamically judging the spatial collision trend in the live area of the equipment, so that the management personnel can use the $3 \mathrm{D}$ scene to realize the remote real-time grasp of on-site operations.

When the positioning coordinates of personnel (or equipment) change, for example, when the on-site personnel (or equipment) deviate from his preset routes, stray into the charged interval, or intrude into the safe distance of charged equipment, the new system will perform collision detection on the area edge of the coordinates of personnel (or equipment) according the virtual operating area (electronic fence) preset in the $3 \mathrm{D}$ scene. When a person (or equipment) enters a dangerous area or goes out of a preset working area by mistake, the system will perform a security risk assessment giving a corresponding real-time alarm. 


\section{Conclusion}

In this paper, we designed and implemented a new 3D visualization safety monitoring and management system for substation, named 3D2S2M based on a 3D reconstruction technology. The new system uses a high-precision 3D lidar scanning equipment to perform an entire station holographic scanning to obtain high-precision laser point cloud data. Based on the point cloud data, a high-accuracy reconstruction of the real scene of the substation is achieved. The new system has the following main functions: virtual 3D space distance measurement is used to replace on-site investigation. Electronic fences are used to set up virtual working areas. High-precision real-time positioning of personnel (or instruments) is achieved. Positioning data and electronic fences are combined to monitor and evaluate the potential risks in the substation and give corresponding real-time warnings. In addition, the new system also realizes the intelligent visualization of operators and equipment in the real 3D scene, which is in line with the development trend of more friendly, more humane and more convenient security monitoring and management in substation, besides the visual management.

\section{Acknowledgment}

This work was supported by China Southern Power Grid Co. Ltd. Science and Technology Program under Grant No. GDKJXM20184850.

\section{References}

[1] X.X. Gao, F. Q. Chen, Design and implementation of a remote positioning system based on GPRS + GPS, China Science and Technology Information, 16 (2015),85-87.

[2] C. Zhao, Study on operation maintenance technology of intelligent substation, Tianjin University, 2011.

[3] X. Li, Study on intelligent reconstruction of substation adapted to ubiquitous IoT, Shandong University, 2019.

[4] L. Zhang, Y. C. Feng, W. T. Qu, Q. K. Shun, Design of aided identification and positioning software for substation operation safety management and control system, Electrotechnical Technology, 4 (2016), 2930.

[5] Y. Gao, Application of 3D digital technology in the substation design, IOP Conference Series Materials Science and Engineering, 782 (2020), 032-066.

[6] J. H. Fang, W. M. Xu, X. F. Zhu, et al., Research and application of intelligent auxiliary control system for substation based on internet of things and 3D visualization technology, Electric Automation, 3 (2012), 71-74.

[7] L. Bai, Research and implementation of 3D digital design of substation, Tianjin: Tianjin University, 2013.

[8] H. Dai, Z. Yang, Z. Xue, et al., Research on key technologies of substation 3D interactive scene simulation based on virtual reality technology, Journal of Physics Conference Series, 1345 (2019), 062036.

[9] X. Suo, C. Hou, L. Sun, et al., 3D reconstruction optimization algorithm based on dynamic clustering in transformer substation[J]. Journal of Computational and Theoretical Nanoence, 2017.

[10] R. D. Wang, Y. Du, X. J. Shen, Status and prospect of 3D modeling methods for substation, North China Electric Power Technology, 2 (2015), 19-23.

[11] J. Quintana, E. Mendoza, 3D virtual models applied in power substation projects, IEEE 15th International Conference on Intelligent System Applications to Power Systems, ISAP'09, Curitiba, Brazil, 2009:1-3.

[12] X. B. Wang, X. D. Zhang, T. He, et al., Digital visual management and monitoring system for 3D virtual substation, Journal of Wuhan University, 6 (2012), 786-791.

[13] X. N. ShiKang, Design and implementation of 3D panoramic view software for substation management system, Xi'an: Xidian University, 2015. 
[14] L. X. Li, Research on classification and identification of substation equipment based on 3D point cloud, Zhengzhou: Zhengzhou University, 2016.

[15] Y. Du, Z. Chen, F. Liu, et al., Three-dimensional real-world deduction simulation of substation operation and risk management and control, Journal of Huaqiao University, 2 (2017), 236-240.

[16] W. H. Hu, Design and implementation of intelligent video surveillance system for unattended substations, Changchun: Jilin University, 2015.

[17] X. Y. Zhou, Research on rapid positioning method and positioning system for mobile personnel in digital substation, Chongqing: Chongqing University, 2013.

[18] X. L. Huang, Research and application of safety protection and monitoring technology for substation operation and maintenance site, Baoding: North China Electric Power University, 2013.

[19] S. K. Yuan, N. N. Gong, Z. Li, et al., Substation personnel positioning and tracking system based on wireless sensor network, System Development and Application, 3 (2013), 50-53.

[20] Y. Zhang, J. H. Hu, C. G. Peng, Research on 3D real-time field operator positioning system for UHV substation based on accurate coordinates, Science \& Technology Innovation Herald, 21 (2017), 1-2.

[21] J. J. Yan, Design of radio frequency identification and location system for substation personnel, Applied Technology, 5 (2015), 94-95.

[22] O. Vozisova, A. Egorov, D. Snegirev, E. Lyuhanov, E. Kochneva, Substation and electric systems equipment 3D laser scanning, 2016 International Symposium on Industrial Electronics (INDEL), Banja Luka, 2016, pp. 1-4.

[23] B. Y. Chen, F. Y. Zhang, Application of cloud model to substation operation risk perception, Journal of Electric Power Systems and Automation, 7 (2019), 23-29. 